\title{
A compromise solution approach for finding common weights in DEA: An improvement to Kao and Hung's approach
}

\author{
Majid Zohrehbandian ${ }^{a, *}$ Ahmad Makui ${ }^{b}$, Alireza Alinezhad ${ }^{c}$ \\ ${ }^{b}$ Department of Mathematics, Islamic Azad University-Karaj Branch, P.O. Box 31485-313, Karaj, Iran. \\ ${ }^{a}$ Department of Industrial Engineering, Iran University of Science \& Technology, Tehran, Iran. \\ ${ }^{c}$ Department of Industrial Engineering, Islamic Azad University-Qazvin Branch, Qazvin, Iran.
}

\begin{abstract}
Data envelopment analysis (DEA) is the leading technique for measuring the relative efficiency of decision making units (DMUs) on the basis of multiple inputs and multiple outputs. In this technique, the weights for inputs and outputs are estimated in the best advantage for each unit so as to maximize its relative efficiency. But, this flexibility in selecting the weights deters the comparison among DMUs on a common base. For dealing with this difficulty, Kao and Hung (2005) proposed a compromise solution approach for generating common weights under the DEA framework. The proposed MCDM model was derived from the original non-linear DEA model. This paper presents an improvement to Kao and Hung's approach by means of introducing an MCDM model which is derived from a new linear DEA model.
\end{abstract}

Keywords: MOLP, Compromise solution, DEA, Efficiency, Ranking, Weight restrictions.

\footnotetext{
${ }^{*}$ Corresponding Author. E-mail Address: zohrebandian@yahoo.com
} 


\section{Introduction}

Data envelopment analysis (DEA) is a method of evaluating relative performance of a group of similar units, called decision making units (DMU). DMUs are essentially perform the same task using similar multiple inputs to produce similar multiple outputs. DEA gives a measure of efficiency, which is essentially defined as a ratio of weighted outputs to weighted inputs. Charnes et al's (1978) idea is to define the efficiency measure by assigning to each unit the most favorable weights as long as the efficiency scores of all DMUs calculated from the same set of weights, do not exceed one.

As the methods of DEA are run separately for each DMU, the set of weights will typically be different for the various DMUs, and in some cases, this is unacceptable that the same factor is accorded widely differing weights. Likewise, the problem of allowing total flexibility of the weights is that the values of the weights obtained by solving the unrestricted DEA program are often in contradiction to prior views or additional available information. For dealing with this difficulty, the concept of weight restrictions and value judgements were introduced in DEA to curb the complete freedom of variation of weights allowed by the original DEA models; see Allen et al. (1997), Chapparo et al. (1997), Podinovski (2001), Podinovski and Athanassopoulos (1998), Wong and Beasley (1990).

This flexibility in selecting the weights, on the other hand, deters the comparison among DMUs on a common base. A possible answer to this difficulty lies in the specification of a common set of weights, which was first introduced by Roll et al. (1991). Research about the idea of common weights has developed gradually in recent years. Recently, Kao and Hung (2005) proposed a compromise solution approach to generate a common set of weights for all DMUs which are able to produce a vector of efficiency scores closest to the efficiency scores calculated from the standard DEA model (ideal solution). Some of the other studies in this field are Cook and Kress (1990), Doyle and Green (1994), Jahanshahloo et al. (2005), Karsak and Ahiska (2005), Roll and Golany (1993).

The proposed MCDM model in Kao and Hung (2005) was derived from the original fractional DEA model which resulted a non-linear model. The aim of this study is improvement of Kao and Hung's approach to produce a linear model with high correlation between its results with that obtained by Kao and Hung (2005).

The plan for the rest of this paper is as follows. In section 2 we present a brief discussion about DEA models and the multiple objective decision making (MODM) problems. The mathematical 
foundation of our method for finding a common set of weights is discussed in Section 3. Numerical example is presented in section 4 and finally, section 5 draws the conclusive remarks.

\section{DEA and MODM Preliminaries}

Thirty years after the publication of the founding paper of Charnes et al. (1978), DEA can safely be considered as one of the recent success stories in operations research and several hundreds of papers have been published since then. Interestingly, Charnes and Cooper have also had a significant impact on the development of multiple objective decision making through the development of goal programming (GP); Charnes and Cooper (1961). Although Charnes and Cooper have played a significant role in the development of DEA and MODM, researchers in these two camps have generally not paid much attention to research performed in the other camp. Some works on the interactions between MODM and DEA, are as follows: Bouyssou (1999), Estellita et al. (2004), Giokas (1997), Golany (1988), Joro et al. (1998), Stewart (1996), Xiao and Reeves (1999).

\subsection{Data Envelopment Analysis}

Consider $\mathrm{n}$ production units, or DMUs, each of which consume varying amount of $\mathrm{m}$ inputs to produce s outputs. Suppose $\mathrm{x}_{i j} \geq 0$ denotes the amount consumed of the i-th input and $\mathrm{y}_{r j} \geq 0$ denotes the amount produced of the r-th output by the j-th decision making unit. Then, the original model, for measuring the efficiency of unit 'p', was a fractional linear program as follows:

$$
\begin{array}{rll}
\operatorname{Max} & \frac{\sum_{r=1}^{s} u_{r} y_{r p}}{\sum_{i=1}^{m} v_{i} x_{i p}} \\
\text { s.t. } & \frac{\sum_{r=1}^{s} u_{r} y_{r j}}{\sum_{i=1}^{m} v_{i} x_{i j}} \leq 1 & j=1, \ldots, n \\
& v_{i}, u_{r} \geq 0 & i=1, \ldots, m \quad r=1, \ldots, s
\end{array}
$$

We can transform the above model to a linear program by setting the denominator in the objective function equal to an arbitrary constant (e.g. unity) and maximizing the numerator. The obtained model is as follows:

$$
\begin{array}{cc}
\left.C C R_{m}\right) & \sum_{r=1}^{s} u_{r} y_{r p} \\
\text { s.t. } & \sum_{r=1}^{s} u_{r} y_{r j}-\sum_{i=1}^{m} v_{i} x_{i j} \leq 0 \quad j=1, \ldots, n \\
\sum_{i=1}^{m} v_{i} x_{i p}=1
\end{array}
$$




$$
v_{i}, u_{r} \geq 0 \quad i=1, \ldots, m \quad r=1, \ldots, s
$$

The above model is called the input oriented CCR multiplier model. The dual problem will also be used afterwards and this is called the input oriented CCR envelopment model:

$$
\begin{array}{rcc}
\left.C C R_{e}\right) & \text { Min } & \theta_{p} \\
\text { s.t. } & \sum_{j=1}^{n} \lambda_{j} x_{i j}-\theta_{p} x_{i p} \leq 0 & i=1, \ldots, m \\
& \sum_{j=1}^{n} \lambda_{j} y_{r j} \geq y_{r p} & r=1, \ldots, s \\
\lambda_{j} \geq 0 & j=1, \ldots, n
\end{array}
$$

$\mathrm{DMU}_{p}$ is called (weakly) efficient if and only if the objective function values associated with the optimal solutions to the above problems are one; otherwise it is inefficient.

Lemma 1 If $\theta_{p}^{*}$ be the optimum solution of model $(3)$, then $\left(\widehat{x_{p}}, \widehat{y_{p}}\right)=\left(\theta_{p}^{*} x_{p}, y_{p}\right)$, called the projection of $\mathrm{DMU}_{p}$ on the efficient frontier, is an efficient (virtual) DMU.

Lemma $2 \mathrm{DMU}_{p}$ is efficient if and only if there exists a nonnegative coefficient $(\mathrm{u}, \mathrm{v}) \in R^{s} \times R^{m}$ associated to the gradient vector of a supporting hyperplane (a hyperplane passing through one of the (extreme) points of the production possibility set (PPS) and the PPS lies completely in one of the two halfspaces into which this hyperplane divides the space $\left.\Re^{m+s}\right)$ where $\sum_{r=1}^{s} u_{r} y_{r p}-\sum_{i=1}^{m} v_{i} x_{i p}=0$.

\subsection{Multiple Objective Decision Making}

MODM problem can be written in the general form as follows:

$$
\begin{array}{cc}
\operatorname{Max} & F(x)=\left\{f_{1}(x), \cdots, f_{k}(x)\right\} \\
\text { s.t. } & x \in X=\left\{x \mid g_{i}(x) \leq 0, i=1, \ldots, m\right\}
\end{array}
$$

Where $\mathrm{x} \in R^{n}$. Against the concept of an optimal solution in single objective mathematical programming problems, in MODM an efficient solution is introduced as follows:

Definition $\mathrm{x}^{*} \in X$ is an efficient solution (or nondominated solution) if and only if there does not exist another $\mathrm{x} \in X$ such that $\forall j: f_{j}(x) \geq f_{j}\left(x^{*}\right)$ and $\exists j: f_{j}(x)>f_{j}\left(x^{*}\right)$.

In order to solve the above problem (identifying the efficient solutions), there are many different methods in the literature. One of these methods is the compromise solution approach. Suppose 
$f_{j}^{*}(x) \quad j=1, \cdots, k$, are the optimum values of the following models:

$$
\begin{array}{cc}
\operatorname{Max} & f_{j}(x), \quad j=1, \cdots, k \\
\text { s.t. } & x \in X
\end{array}
$$

Then an efficient solution is defined as the one which minimizes the deviations from these optimum values (ideal solution) as follows:

$$
\begin{array}{cc}
\text { Min } & D_{p}=\left\{\sum_{j=1}^{k}\left[\frac{f_{j}^{*}(x)-f_{j}(x)}{f_{j}^{*}(x)}\right]^{p}\right\}^{\frac{1}{p}}, \quad p \geq 1 \\
\text { s.t. } & x \in X
\end{array}
$$

Where the value of $\mathrm{p}$ is based upon the utility function of the DM. In the above Model, for the smallest value of $\mathrm{p}=1$, every deviation is being weighted equally. As $\mathrm{p}$ increases, more weights are given to the larger deviations. Ultimately, the largest deviation completely dominates when $\mathrm{p}=\infty$. However, there are three values of $\mathrm{p}$, viz., $\mathrm{p}=1,2$, and $\infty$, which have special mathematical properties and are worthy of consideration.

\section{A Method for Finding a Common Set of Weights}

In DEA for calculating the efficiency of different DMUs, different set of weights are obtained, which seems to be unacceptable in reality. Kornbluth (1991) noticed that the DEA model could be expressed as a multi-objective linear fractional programming problem. The objective function of the model is the same as in the CCR model (1), but applied to maximize efficiency of all DMUs, instead of one at a time, and the restrictions remaining unchanged. However, the proposed model was nonlinear. Some other methods also proposed in the literature which were based on Kornbluth's approach. One of them is the compromise solution approach of Kao and Hung (2005). Below, we present a brief discussion of their approach.

The optimal objective value of model (1), denoted by $\mathrm{E}_{p}^{*}$, is the best attainable efficiency level for $\mathrm{DMU}_{p}$. Any other set of weights would result in an efficiency score which is less than or equal to $\mathrm{E}_{p}^{*}$. In generating a common set of weights for all DMUs, Kao and Hung (2005) consider $E^{*}=\left(E_{1}^{*}, \ldots, E_{n}^{*}\right)$ as the target, or ideal solution, to achieve. They want the efficiency vector $E(u, v)=\left(E_{1}(u, v), \ldots, E_{n}(u, v)\right)$ calculated from the common weights $(\mathrm{u}, \mathrm{v})$ to be closest to the ideal solution $\mathrm{E}^{*}$. To determine the degree of closeness between $\mathrm{E}(\mathrm{u}, \mathrm{v})$ an $\mathrm{E}^{*}$, a generalized family of distance measures was applied: 


$$
D_{p}(E(u, v))=\left[\sum_{j=1}^{n}\left(E_{j}^{*}-E_{j}(u, v)\right)^{p}\right]^{\frac{1}{p}}, \quad p \geq 1
$$

where $\mathrm{p}$ represents the distance parameter. Then, the common weights $(\mathrm{u}, \mathrm{v})$ and the associated efficiency scores $E_{j}(u, v) j=1, \cdots, n$, solved from a mathematical program, called the compromise solution with parameter $\mathrm{p}$, which is equivalent to a linearly constrained nonlinear program.

Now, suppose $\theta_{j}^{*}, \mathrm{j}=1, \ldots, \mathrm{n}$, are the optimum values obtained from the model $\mathrm{CCR}_{e}$, when $\mathrm{DMU}_{j}$ is under consideration and $\left(\widehat{x_{j}}, \widehat{y_{j}}\right)=\left(\theta_{j}^{*} x_{j}, y_{j}\right)$, which is an efficient DMU according to lemma 1. In this section, we present an improvement to Kao and Hung's approach by introducing a multiple objective linear programming (MOLP) for finding common weights in DEA. But, firstly, the following model is introduced to find efficiency value of $\mathrm{DMU}_{p}$ which has the same results as the CCR multiplier model. However, it has some advantages compared to model (2) that will be discussed later on.

$$
\begin{array}{cl}
\operatorname{Max} & \sum_{r=1}^{s} u_{r} \widehat{y_{r p}}-\sum_{i=1}^{m} v_{i} \widehat{x_{i p}} \\
\text { s.t. } & \sum_{r=1}^{s} u_{r} \widehat{y_{r j}}-\sum_{i=1}^{m} v_{i} \widehat{x_{i j}} \leq 0 \quad j=1, \ldots, n \\
& \sum_{r=1}^{s} u_{r}+\sum_{i=1}^{m} v_{i}=1 \\
v_{i}, u_{r} \geq 0 & i=1, \ldots, m \quad r=1, \ldots, s
\end{array}
$$

Here, the constraint $\sum_{r=1}^{s} u_{r}+\sum_{i=1}^{m} v_{i}=1$ is superfluous. However, it corresponds to the constraint $\sum_{i=1}^{m} v_{i} x_{i p}=1$ in model (2). Moreover, its effect is to have normalized weights $\mathrm{u}$ and $\mathrm{v}$ (uniqueness of optimal solution). The dual problem of model (7) is as follows:

$$
\begin{array}{ccc}
\text { Min } & z & \\
\text { s.t. } & \sum_{j=1}^{n} \lambda_{j} \widehat{x_{i j}}-z \leq \widehat{x_{i p}} & i=1, \ldots, m \\
& \sum_{j=1}^{n} \lambda_{j} \widehat{y_{r j}}+z \geq \widehat{y_{r p}} & r=1, \ldots, s \\
\lambda_{j} \geq 0 & j=1, \ldots, n
\end{array}
$$

Here, due to the fact that $\left(\widehat{x_{p}}, \widehat{y_{p}}\right)$ is an efficient DMU (it is not possible to improve all of inputs and outputs of an efficient DMU), we have $z^{*}=0$. In other words, the optimum value of model (7) is zero.

Theorem 1 For optimal solution of model (7), say $\left(u^{*}, v^{*}\right)$, we have: $\frac{\sum_{r=1}^{s} u_{r}^{*} y_{r p}}{\sum_{i=1}^{m} v_{i}^{*} x_{i p}}=\theta_{p}^{*}$.

Proof The production possibility set produced by $\left(\widehat{x_{j}}, \widehat{y_{j}}\right)=\left(\theta_{j}^{*} x_{j}, y_{j}\right) \mathrm{j}=1, \cdots, \mathrm{n}$, is the same as 
original PPS. Furthermore, since $\left(\theta_{p}^{*} x_{p}, y_{p}\right)$ is input oriented projection of $\mathrm{DMU}_{p}$ on the efficient frontier, according to foregoing lemmas (1 and 2) it is efficient and there is a supporting hyperplane of PPS at $\left(\widehat{x_{p}}, \widehat{y_{p}}\right)=\left(\theta_{p}^{*} x_{p}, y_{p}\right)$ which will result $\frac{\sum_{r=1}^{s} u_{r}^{*} y_{r p}}{\sum_{i=1}^{m} v_{i}^{*} x_{i p}}=\theta_{p}^{*} \square$

Suppose $\left(\widehat{x_{j}}, \widehat{y_{j}}\right)=\left(\theta_{j}^{*} x_{j}, y_{j}\right) j=1, \cdots, n$. Then, according to the above model and the proposed approach by Kornbluth (1991), the idea behind the identification of the common weights is formulated as the following MOLP problem. The objective function of the model is the same as in the model (7), but applied for all DMUs, instead of one at a time, and the restrictions remaining unchanged.

$$
\begin{array}{ccc}
\operatorname{Max}\left[\sum_{r=1}^{s} u_{r} \widehat{y_{r 1}}-\sum_{i=1}^{m} v_{i} \widehat{x_{i 1}}, \cdots, \sum_{r=1}^{s} u_{r} \widehat{y_{r n}}-\sum_{i=1}^{m} v_{i} \widehat{x_{i n}}\right] & \\
\text { s.t. } & \sum_{r=1}^{s} u_{r} \widehat{y_{r j}}-\sum_{i=1}^{m} v_{i} \widehat{x_{i j}} \leq 0 & j=1, \ldots, n \\
& \sum_{r=1}^{s} u_{r}+\sum_{i=1}^{m} v_{i}=1 & i=1, \ldots, m \\
v_{i}, u_{r} \geq 0 &
\end{array}
$$

Furthermore, in order to solve the above MOLP model, we use the compromise solution approach to produce a vector of deviation scores closest to the scores calculated from the model (7). Hence, a vector of zero scores is considered as an ideal solution. By using the generalized family of distance measures as follows:

$$
D_{p}(u, v)=\left[\sum_{j=1}^{n}\left(0-\left(\sum_{r=1}^{s} u_{r} \widehat{y_{r j}}-\sum_{i=1}^{m} v_{i} \widehat{x_{i j}}\right)\right)^{p}\right]^{\frac{1}{p}}, \quad p \geq 1
$$

where $\mathrm{p}$ represents the distance parameter, the following mathematical programming, called compromise solution with parameter $\mathrm{p}$, is produced.

$$
\begin{array}{ccc}
\text { Min } & D_{p}(u, v)=\left[\sum_{j=1}^{n}\left(-\sum_{r=1}^{s} u_{r} \widehat{y_{r j}}+\sum_{i=1}^{m} v_{i} \widehat{x_{i j}}\right)^{p}\right]^{\frac{1}{p}}, p \geq 1 & \\
\text { s.t. } & \sum_{r=1}^{s} u_{r} \widehat{y_{r j}}-\sum_{i=1}^{m} v_{i} \widehat{x_{i j}} \leq 0 & j=1, \ldots, n \\
& \sum_{r=1}^{s} u_{r}+\sum_{i=1}^{m} v_{i}=1 & \\
v_{i}, u_{r} \geq 0 & i=1, \ldots, m
\end{array}
$$

Although model (10) is equivalent to a linearly constrained nonlinear program, it is completely linear for $\mathrm{p}=1$ and $\infty$. Therefore, for $\mathrm{p}=1$ and $\infty$ it is solved easier than proposed model by Kao and Hung (2005) which was completely nonlinear. 
The duals of model (10) for the case $\mathrm{p}=1, \infty$ are as follows:

$$
\begin{array}{rrr}
\operatorname{Max} & z & \\
\text { s.t. } & \sum_{j=1}^{n} \lambda_{j} \widehat{x_{i j}} \leq \sum_{j=1}^{n} \widehat{x_{i j}}-z \quad i=1, \ldots, m \\
& \sum_{j=1}^{n} \lambda_{j} \widehat{y_{r j}} \geq \sum_{j=1}^{n} \widehat{y_{r j}}+z & r=1, \ldots, s \\
\lambda_{j} \geq 0 & j=1, \ldots, n \\
\operatorname{Max} & \sum_{j=1}^{n} \lambda_{j} \widehat{x_{i j}} \leq \sum_{j=1}^{n} \beta_{j} \widehat{x_{i j}}-z & i=1, \ldots, m \\
\text { s.t. } & \sum_{j=1}^{n} \lambda_{j} \widehat{y_{r j}} \geq \sum_{j=1}^{n} \beta_{j} \widehat{y_{r j}}+z & \\
& \sum_{j=1}^{n} \beta_{j}=1 & \\
\lambda_{j}, \beta_{j} \geq 0 & j=1, \ldots, s
\end{array}
$$

Here, model (11) corresponds to the radius of stability model, see Cooper et al (2001), of a unit with inputs and outputs equal to $\left(\sum_{j=1}^{n} \widehat{x_{j}}, \sum_{j=1}^{n} \widehat{y_{j}}\right)=\left(\sum_{j=1}^{n} \theta_{j}^{*} x_{j}, \sum_{j=1}^{n} y_{j}\right)$, which corresponds to the sum of all the radially input projected units.

With respect to the dual of model (10) for the case $\mathrm{p}=\infty$, it would be slightly different. In this case, model (12), the unit for which the radius of stability is computed is determined by the own model among those that belong to the convex hull of the radially input projected units $\left(\widehat{x_{j}}, \widehat{y_{j}}\right)=\left(\theta_{j}^{*} x_{j}, y_{j}\right) j=1, \cdots, n$. The solution to the model will be the radius of stability of the furthest point (using Tchebycheff metric) within the convex hull to the efficient frontier.

However, solving the model (10) give us a common set of weights and then efficiency score of $\mathrm{DMU}_{j}, \mathrm{j}=1, \ldots, \mathrm{n}$, can be obtained by using these common weights as $\frac{\sum_{r=1}^{s} u_{r}^{*} y_{r j}}{\sum_{i=1}^{m} v_{i}^{*} x_{i j}}$. If for $\left(u^{*}, v^{*}\right)$ we have $\frac{\sum_{r=1}^{s} u_{r}^{*} y_{r p}}{\sum_{i=1}^{m} v_{i}^{*} x_{i p}}=1$, then $\mathrm{DMU}_{p}$ is called efficient. Furthermore, by defining the set $\mathrm{A}=\{\mathrm{j}$ : $\mathrm{DMU}_{j}$ is efficient in model (10)\}, and using the same approach as in Jahanshahloo et al. (2005) a complete ranking of DMUs will be obtained.

Theorem 2 Suppose that $\mathrm{DMU}_{p}$ is indicated efficient by model (10). Then, it is efficient in an input oriented CCR model.

Proof According to the first inequalities we have: $\frac{\sum_{r=1}^{s} u_{r}^{*} y_{r p}}{\sum_{i=1}^{m} v_{i}^{*} x_{i p}} \leq \theta_{p}^{*} \leq 1$. Therefore, if $\frac{\sum_{r=1}^{s} u_{r}^{*} y_{r p}}{\sum_{i=1}^{m} v_{i}^{*} x_{i p}}=1$ 
then $\theta_{p}^{*}=1$ and $\mathrm{DMU}_{p}$ is CCR efficient $\square$

Theorem 3 There is a $\mathrm{DMU}_{j}, \mathrm{j}=1, \ldots, \mathrm{n}$ for which model (10) characterize it as an efficient DMU. Proof Suppose $\mathrm{p}=1$ or $\infty$ in model (10). There is a $\mathrm{DMU}_{p}, \mathrm{p} \in\{1, \cdots, n\}$ for which the first inequality in (10) is binding. Because, if it is not the case, there is a sufficiently small value $\varepsilon>0$ where $(\bar{u}, \bar{v})=\left(u^{*}+[\varepsilon, 0, \ldots, 0]_{1 \times s}^{T}, v^{*}-[\varepsilon, 0, \ldots, 0]_{1 \times m}^{T}\right)$ satisfy the set of restrictions in (10). On the other hand, the value of $D_{p}(\bar{u}, \bar{v})$ is smaller than $D_{p}\left(u^{*}, v^{*}\right)$ and this is a contradiction.

Therefore, there is a $\mathrm{DMU}_{p}, \mathrm{p} \in\{1, \cdots, n\}$ for which we have: $\sum_{r=1}^{s} u_{r}^{*} \widehat{y_{r p}}-\sum_{i=1}^{m} v_{i}^{*} \widehat{x_{i p}}=0$. Such a $\left(u^{*}, v^{*}\right)$ is associated to the gradient vector of a supporting hyperplane. Furthermore, this supporting hyperplane must support the PPS at some extreme efficient DMUs. Therefore, such extreme efficient DMUs are indicated efficient by the model (10) $\square$

Roll et al. (1991) and Golany and Yu (1995) indicate that a general requirement for the common set of weights is that at least one DMU must attain efficiency 1 with the common weights. If there is no DMU with efficiency score 1, then it is obvious that the efficiencies are under-estimated in the sense of relative comparison. In this sense, the efficiency scores obtained from our proposed method are not under-estimated and satisfied the general requirement.

\section{Numerical Example}

To illustrate the idea of the proposed approach, an example from Kao and Hung (2005) is utilized. In that example, there are 17 DMUs with four inputs and three outputs.

Table (1) contains the common set of weights generated by proposed method in this paper (for $\mathrm{p}=1$, and $\infty$ ), respect to inputs and outputs. Furthermore, table (2) shows the efficiency scores of DMUs calculated from the CCR Model, the compromise solution approach by Kao and Hung (2005), and the compromise solution approach in this paper, respectively.

The CCR efficiency scores are the highest values that the DMUs can attain, and there are nine efficient units which cannot be differentiated. Regarding the compromise solution approach by Kao and Hung (2005), two values of p, viz., 1, and $\infty$, have been considered and the results are referred to as $\mathrm{MAD}_{1}$, and $\mathrm{MAX}_{1}$. Likewise, Regarding to the compromise solution approach in this paper, two values of $\mathrm{p}$, viz., 1 , and $\infty$, have been considered and the results are referred to as $\mathrm{MAD}_{2}$, and $\mathrm{MAX}_{2}$.

Note that, it is inappropriate to say which weights from these four common weights are correct and which are not. They are different sets of weights due to the fact that they are obtained from 
different viewpoints. But in general, these common weights are more informative than those of the CCR model. They not only differentiate the efficient units, but also assess all of the DMUs on the same scale.

We can also use correlation between the set of efficiency scores of the two compromise solution approaches obtained for two values of $\mathrm{p}$, viz., 1 , and $\infty$, for verifying the results of the approach. Like the Pearson product moment correlation coefficient, Spearman's $\rho$ is a measure of the relationship between two variables. However, Spearman's $\rho$ is calculated on ranked data. For calculating Spearman's $\rho$ we can use the below formulation where $d_{i}$ is the difference between ranks for the same observation (DMU) and $\mathrm{n}$ is the number of DMUs.

$$
r_{s}=1-\frac{6 \sum_{i=1}^{n} d_{i}^{2}}{n\left(n^{2}-1\right)}
$$

Likewise, we can compute the Pearson's correlation on the columns of ranked data. The results of this formulation is too close to the exact Spearman's $\rho$. In this formulation $x_{j}$ and $y_{j}$ are the ranks for the same $\mathrm{DMU}_{j} j=1, \ldots, n$.

$$
r=\frac{\sum x_{i} y_{i}-m \bar{x} \bar{y}}{\sqrt{\sum x_{i}^{2}-n \bar{x}^{2}} \sqrt{\sum y_{i}^{2}-n \bar{y}^{2}}}
$$

Empirically, in this example the Spearman's correlation between the set of efficiency scores of the two compromise solution approaches (Kao and Hung's approach and our approach) obtained for two values of p, viz., 1 , and $\infty$, are 0.963 and 0.914 , respectively. However, use of the proposed approach in this paper has an advantage over the Kao and Hung's approach. Our approach is based on solution of a linear problem but Kao and Hung's approach is based on solution of a nonlinear problem.

\section{Conclusion}

For assessment of all the DMUs on the same scale, this paper examines the application of compromise solution approach for generating common set of weights under the DEA framework. There are other methods in the literature which are also able to generate common weights. A case taken from Kao and Hung (2005) is solved to investigate the differences among these methods and some conclusions are derived.

The proposed approach in this paper is based on solution of a linear problem. Hence, the use of it has an advantage over the general approaches in the literature, which are based on solving nonlinear problems. When weights of the input/output factors are available, efficiency scores can be measured. Moreover, all DMUs can be ranked in terms of a common base. Finally, the proposed 
method, simply and with appropriate modifications, can be generalized to the other DEA models.

\section{References}

[1] Allen R., Athanassopoulos A., Dyson R.G., Thanassoulis E. (1997), "Weights restrictions and value judgements in Data Envelopment Analysis: Evolution, development and future directions," Annals of Operations Research, 73, 13-34.

[2] Bouyssou D. (1999), "Using DEA as a tool for MCDM: some remarks," Journal of the Operational Research Society, 50(9), 974-978.

[3] Chapparo F.P., Jimenez J.S., Smith P. (1997), "On the Role of Weight Restrictions in Data Envelopment Analysis," Journal of Productivity Analysis, 8, 215-230.

[4] Charnes A., Cooper W.W. (1961), "Management Models and Industrial Applications of Linear Programming," John Wiley, New York.

[5] Charnes A., Cooper W.W., Rhodes E. (1978), "Measuring the efficiency of decision making units," European Journal of Operational Research, 2, 429-444.

[6] Cook W.D., Kress M. (1990), "Data envelopment model for aggregating preference ranking," Management Science, 36(11), 1302-1310.

[7] Cooper W.W., Li s., Seiford L.M., Tone K., Thrall R.M., Zhu j. (2001), "Sensitivity and Stability Analysis in DEA: Some Recent Developments," Journal of Productivity Analysis, 15, $217-246$.

[8] Doyle J.R., Green R.H. (1994), "Efficiency and cross-efficiency in DEA: derivatives, meanings and uses," Journal of the Operational Research Society, 45, 567-578.

[9] Estellita Lins M.P., Angulo Meza L.,Moreira da Silva A.C. (2004), "A multi-objective approach to determine alternative targets in data envelopment analysis," Journal of the Operational Research Society, 55, 1090-1101. 
[10] Giokas D. (1997), "The use of goal programming and data envelopment analysis for estimating efficient marginal costs of outputs," Journal of the Operational Research Society, 48(3), 319323.

[11] Golany B. (1988), "An interactive MOLP procedure for the extension of DEA to effectiveness analysis," Journal of the Operational Research Society, 39(8), 725-734.

[12] Golany B., Yu G. (1995), "A goal programming-discriminant function approach to the estimation of an empirical production function based on DEA results," Journal of Productivity Analysis, 6, 171-186.

[13] Jahanshahloo G.R., Memariani A., Lotfi F.H., Rezai H.Z. (2005), "A note on some of DEA models and finding efficiency and complete ranking using common set of weights," Applied Mathematics and Computation, 166, 265-281.

[14] Joro T., Korhonen P., Wallenius J. (1998), "Structural comparison of data envelopment analysis and multiple objective linear programming," Management Science, 44, 962-970.

[15] Kao C., Hung H.T. (2005), "Data envelopment analysis with common weights: the compromise solution approach," Journal of the Operational Research Society, 56, 1196-1203.

[16] Karsak E.E., Ahiska S.S. (2005), "Practical common weight multi-criteria decision-making approach with an improved discriminating power for technology selection," International Journal of Production Research, 43(8), 1537-1554.

[17] Kornbluth J. (1991), "Analysing policy effectiveness using cone restricted data envelopment analysis," Journal of the Operational Research Society, 42, 1097-1104.

[18] Podinovski V.V. (2001), "Validating absolute weights bounds in Data Envelopment Analysis (DEA) models," Journal of the Operational Research Society, 52(2), 221-225.

[19] Podinovski V.V., Athanassopoulos A.D. (1998), "Assessing the relative efficiency of decision making units using DEA models with weight restrictions," Journal of the Operational Research Society, 49(5), 500-508.

[20] Roll Y., Cook W.D., Golany B. (1991), "Controlling factor weights in data envelopment analysis," IIE Transactions, 23(1), 2-9. 
[21] Roll Y., Golany B. (1993), "Alternate methods of treating factor weights in DEA," Omega, 21(1), 99-109.

[22] Stewart T.J. (1996), "Relationships between data envelopment analysis and multicriteria decision-analysis," Journal of the Operational Research Society, 47(5), 654-665.

[23] Wong Y., Beasley J.E. (1990), "Restricting weight flexibility in data envelopment analysis," Journal of the Operational Research Society, 41(9), 829-835.

[24] Xiao Bai L., Reeves G.R. (1999), "A multiple criteria approach to data envelopment analysis," European Journal of Operational Research, 115, 507-517 


\begin{tabular}{|c|c|c|c|c|c|c|c|}
\hline Method & $\mathrm{v}_{1}$ & $\mathrm{v}_{2}$ & $\mathrm{v}_{3}$ & $\mathrm{v}_{4}$ & $\mathrm{u}_{1}$ & $\mathrm{u}_{2}$ & $\mathrm{u}_{3}$ \\
\hline $\mathrm{p}=1$ & 0.20026 & 0.34628 & 0.00010 & 0.03421 & 0.06658 & 0.35022 & 0.00236 \\
\hline $\mathrm{p}=\infty$ & 0.000100 & 0.404158 & 0.097841 & 0.074547 & 0.075495 & 0.346710 & 0.001149 \\
\hline
\end{tabular}

Table 1. Common weights generated from the proposed compromise solution approach.

\begin{tabular}{|c|c|cc|cc|}
\hline DMUs & CCR & MAD $_{1}^{*}$ & $\mathrm{MAX}_{1}^{*}$ & $\mathrm{MAD}_{2}$ & $\mathrm{MAX}_{2}$ \\
\hline 1 & $1.0000(1)$ & $1.0000(1)$ & $1.0000(1)$ & $1.0000(1)$ & $1.0000(1)$ \\
2 & $1.0000(1)$ & $1.0000(1)$ & $1.0000(1)$ & $1.0000(1)$ & $1.0000(1)$ \\
3 & $1.0000(1)$ & $1.0000(1)$ & $0.7231(11)$ & $1.0000(1)$ & $0.7433(9)$ \\
4 & $1.0000(1)$ & $1.0000(1)$ & $0.8984(4)$ & $1.0000(1)$ & $0.8537(6)$ \\
5 & $1.0000(1)$ & $0.9747(5)$ & $1.0000(1)$ & $1.0000(1)$ & $0.9442(4)$ \\
6 & $1.0000(1)$ & $0.8524(9)$ & $0.8692(7)$ & $0.9654(6)$ & $0.8351(7)$ \\
7 & $1.0000(1)$ & $0.9244(6)$ & $0.7432(9)$ & $0.8743(8)$ & $0.7109(12)$ \\
8 & $1.0000(1)$ & $0.8954(7)$ & $0.8939(5)$ & $0.8469(9)$ & $0.9619(3)$ \\
9 & $1.0000(1)$ & $0.6619(14)$ & $0.7230(12)$ & $0.6783(13)$ & $0.6494(15)$ \\
10 & $0.9403(10)$ & $0.8721(8)$ & $0.8761(6)$ & $0.8779(7)$ & $0.8995(5)$ \\
11 & $0.9346(11)$ & $0.6398(15)$ & $0.6577(13)$ & $0.6526(15)$ & $0.7456(8)$ \\
12 & $0.8290(12)$ & $0.7456(10)$ & $0.7594(8)$ & $0.7175(11)$ & $0.7265(11)$ \\
13 & $0.7997(13)$ & $0.6229(17)$ & $0.6453(14)$ & $0.6227(16)$ & $0.6919(13)$ \\
14 & $0.7733(14)$ & $0.7140(12)$ & $0.7406(10)$ & $0.7126(12)$ & $0.7428(10)$ \\
15 & $0.7627(15)$ & $0.7245(11)$ & $0.6410(15)$ & $0.7215(10)$ & $0.6691(14)$ \\
16 & $0.7435(16)$ & $0.6996(13)$ & $0.4665(17)$ & $0.6696(14)$ & $0.5554(17)$ \\
17 & $0.6873(17)$ & $0.6310(16)$ & $0.5908(16)$ & $0.5925(17)$ & $0.5667(16)$ \\
\hline Average & 0.910 & 0.821 & 0.778 & 0.819 & 0.782 \\
\hline
\end{tabular}

Table 2. Efficiency scores and the associated rankings (in parentheses) calculated from the CCR ratio model and different methods of common weights.

* Results obtained from Kao and Hung (2005). 